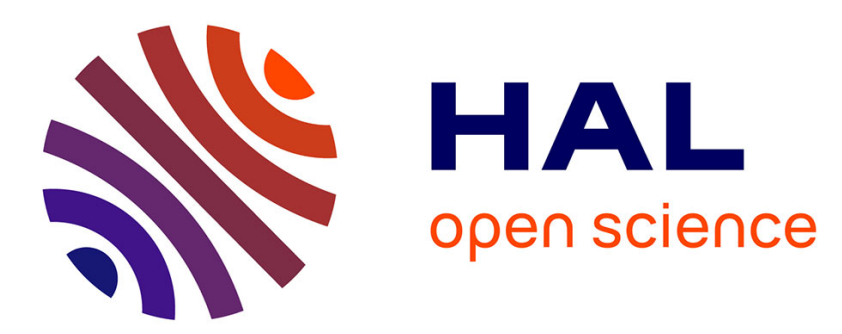

\title{
Interplay between turgor pressure and plasmodesmata during plant development
}

\author{
Valeria Hernández-Hernández, Mariana Benítez, Arezki Boudaoud
}

\section{To cite this version:}

Valeria Hernández-Hernández, Mariana Benítez, Arezki Boudaoud. Interplay between turgor pressure and plasmodesmata during plant development. Journal of Experimental Botany, 2020, 71 (3), pp.768777. 10.1093/jxb/erz434. hal-03153187

\section{HAL Id: hal-03153187 \\ https://hal.inrae.fr/hal-03153187}

Submitted on 7 Jun 2021

HAL is a multi-disciplinary open access archive for the deposit and dissemination of scientific research documents, whether they are published or not. The documents may come from teaching and research institutions in France or abroad, or from public or private research centers.
L'archive ouverte pluridisciplinaire HAL, est destinée au dépôt et à la diffusion de documents scientifiques de niveau recherche, publiés ou non, émanant des établissements d'enseignement et de recherche français ou étrangers, des laboratoires publics ou privés.

\section{(c)(1)}

Distributed under a Creative Commons Attribution| 4.0 International License 


\title{
Interplay between turgor pressure and plasmodesmata during plant development
}

\author{
Valeria Hernández-Hernández ${ }^{1}$, Mariana Benítez ${ }^{2}$ and Arezki Boudaoud ${ }^{1, *}$ \\ ${ }^{1}$ Laboratoire Reproduction et Développement des Plantes, Université de Lyon, ENS de Lyon, UCB Lyon 1, CNRS, INRA, Lyon, France \\ ${ }^{2}$ Laboratorio Nacional de Ciencias de la Sostenibilidad, Instituto de Ecología \& Centro de Ciencias de la Complejidad, Universidad \\ Nacional Autónoma de México, Mexico City, Mexico \\ * Correspondence: arezki.boudaoud@ens-lyon.fr
}

Received 16 July 2019; Editorial decision 9 September 2019; Accepted 9 September 2019

Editor: Christine Raines, University of Essex, UK

\begin{abstract}
Plasmodesmata traverse cell walls, generating connections between neighboring cells. They allow intercellular movement of molecules such as transcription factors, hormones, and sugars, and thus create a symplasmic continuity within a tissue. One important factor that determines plasmodesmal permeability is their aperture, which is regulated during developmental and physiological processes. Regulation of aperture has been shown to affect developmental events such as vascular differentiation in the root, initiation of lateral roots, or transition to flowering. Extensive research has unraveled molecular factors involved in the regulation of plasmodesmal permeability. Nevertheless, many plant developmental processes appear to involve feedbacks mediated by mechanical forces, raising the question of whether mechanical forces and plasmodesmal permeability affect each other. Here, we review experimental data on how one of these forces, turgor pressure, and plasmodesmal permeability may mutually influence each other during plant development, and we discuss the questions raised by these data. Addressing such questions will improve our knowledge of how cellular patterns emerge during development, shedding light on the evolution of complex multicellular plants.
\end{abstract}

Keywords: Hydraulic conductivity, mechanical stress, permeability, plasmodesmata, turgor pressure.

\section{Introduction}

Plasmodesmata are channels that traverse the cell walls and create symplasmic continuity. They have been shown to play a key role in the mobilization of different types of molecules such as hormones, sucrose, transcription factors, and viruses (De Storme and Geelen, 2014; Sager and Lee, 2014; Han and Kim, 2016). In opposition to animal development, which relies partly on cell migration to establish cellular patterns, plant cells are encased within the cell wall and therefore symplasmic connectivity seems pivotal to establishing cell types. Studies have proven that regulation of plasmodesmal permeability helps establish patterns of cell identity during plant development (De
Storme and Geelen, 2014; Sager and Lee, 2014). Accordingly, it has been proposed that plasmodesmata may have had a key role in the evolution of multicellular forms in plants (Lucas and Lee, 2004; Benítez et al., 2018).

One interesting feature of plasmodesmata is that their permeability can vary among different types of cells, organs, and throughout developmental stages. Changes in symplasmic connectivity have been correlated to changes in size of the plasmodesmal pore and in plasmodesmal number. Variations in symplasmic connectivity have been observed under a wide range of phenomena such as pathogen infection (Lee et al., 
2011), mechanical stimulation (Jaffe et al., 1985), transition to dormancy (Rinne and van der Schoot, 1998), flower initiation (Gisel et al., 1999), the transition of leaves from sink to source (Oparka et al., 1999), and as a response to manipulation of mechanical forces (Oparka and Prior, 1992). Interestingly, the interaction between mechanical forces and molecular processes is important for the development of plants (Hamant and Traas, 2009; Moulia, 2013).

Plant growth and morphogenesis involve cell expansion, which is driven by turgor pressure that, in turn, depends on the supply of solutes. The amount of solutes within the cell can vary as a result, for example, of their transport by membrane transporters, the enzymatic activity of proteins that synthesize de novo solute molecules, and intercellular transport through plasmodesmata. In this Review, we put forward the hypothesis that turgor pressure might also regulate plasmodesmal permeability based on previous observations that plasmodesmal permeability can respond to changes in turgor (Oparka and Prior, 1992) and that osmotic treatments are related to changes in the size of the plasmodesmal channel (Schulz, 1995). We discuss the evidence that suggests the existence of feedback interactions between turgor pressure and plasmodesmal permeability. Beforehand, we explain the concepts behind turgor pressure and briefly review the role of plasmodesmata. Finally, we discuss the potential relevance of these bidirectional interactions and identify some of the related open questions.

\section{Turgor pressure in the cell}

Small molecules such as sucrose can move through plasmodesmata (Schulz, 1995) and patterns of unloading in sink tissues have been correlated with patterns of cell expansion in Pisum sativum stems (Schmalstig and Cosgrove, 1990). The amount of solutes such as sucrose, i.e. osmolytes, within the cell is important because they mix with water and thus tend to reduce the free energy of water, that is, the water potential $(\Psi)$.Van't Hoff's equation may be used to estimate the osmotic potential $\left(\Psi_{\pi}\right)$ for low concentrations of solutes, $\Psi_{\pi}=-R T c_{\mathrm{s}}$, where $R$ is the gas constant $\left(8.32 \mathrm{~J} \mathrm{~mol}^{-1} \mathrm{~K}^{-1}\right), T$ is the temperature (given in kelvins), and $c_{\mathrm{s}}$ is the concentration of osmolytes per volume of water, i.e. the osmolarity expressed in moles of solutes per meter cubed of solution (Niklas and Spatz, 2012). Note that osmolarity differs from molarity, which corresponds to the concentration (in moles per meter cubed) before dissolution; for instance, sodium chloride with a molarity of $1 \mathrm{~mol}$ $\mathrm{m}^{-3}$ dissociates into $\mathrm{Na}^{+}$and $\mathrm{Cl}^{-}$ions corresponding to an osmolarity of $2 \mathrm{~mol} \mathrm{~m}^{-3}$, whereas the osmolarity of glucose in solution is equal to its molarity because there is no dissociation. The osmotic potential is negative because dissolution of solutes is thermodynamically more favorable than pure water $(\Psi=0)$. The negative of $\Psi_{\pi}$ is referred to as 'osmotic pressure'. As membranes are selectively permeable (i.e. they allow free movement of water, but not of all solutes), differences in concentration of solutes that do not move freely across the membrane generate a gradient in water potential that drives movement of water into or out of the cell. Two other factors also contribute to water potential: gravity potential $\left(\Psi_{\mathrm{g}}\right)$ and hydrodynamic pressure potential $\left(\Psi_{\mathrm{p}}\right)$ :

$$
\Psi=\Psi_{\mathrm{g}}+\Psi_{\mathrm{p}}+\Psi_{\pi}
$$

Gravity causes water to move downward unless an opposite force acts. The effect of gravity potential depends on height, the density of water, and the acceleration of gravity (Niklas and Spatz, 2012). An upward vertical distance of 10 $\mathrm{m}$ is translated into an increase of $0.1 \mathrm{MPa}$ in water potential. At the cellular scale, gravity is usually neglected because a vertical distance of $1 \mathrm{~m}$ can cause a change in the water potential of $0.01 \mathrm{MPa}$, which is small compared with the contribution of other factors to changes in water potential. The pressure potential is defined as the hydrodynamic pressure of a solution and is generally quantified as a deviation from the ambient pressure. When the hydrodynamic pressure is higher than atmospheric pressure it applies a force on the walls of the container, which is then 'pressurized' (Beauzamy et al., 2014); such pressure is usually called 'turgor pressure'. Negative values (tensional force) of hydrodynamic pressure are achieved under very specific conditions, e.g. in the xylem (Niklas and Spatz, 2012).

Turgor pressure is important for processes like cell expansion (Kroeger et al., 2011), closure and opening of stomata (Buckley, 2005), and transport in the phloem (Münch, 1930; Niklas and Spatz, 2012); developmental changes in turgor have been proposed to be involved in lateral root emergence (Péret et al., 2012; Lucas et al., 2013; Vermeer et al., 2014). Indeed, cellular elongation depends on the balance between turgor pressure and the mechanics of the cell wall. The cell wall is a rigid structure made of cellulose fibers embedded in a matrix of hemicellulose and pectin (Cosgrove, 2005). In normal conditions, the wall is stretched by the protoplast, creating mechanical stress in the load-bearing components of the cell wall that, in turn, compress the protoplast (Cosgrove, 1985; Schopfer, 2006). Turgor pressure is the hydrostatic internal force that acts in opposition to the compression by the wall. When the cell wall loosens (or relaxes) turgor drops and this, in turn, reduces the internal water potential, which then drives water influx and cell elongation (Schopfer, 2006).

In a simple system, water will flow through the membrane from the compartment with the highest water potential towards that with the lowest water potential, tending to equilibrate this difference. In a plant cell, the plasma membrane is often idealized as a semi-permeable membrane, though it partially enables the movement of many osmolytes (Beauzamy et al., 2014). Processes that regulate the internal concentration of osmolytes can also change the water potential. For example, the conversion of glucose into fructose and sucrose by vacuolar invertases (Wang et al., 2010). Water absorption tends to dilute osmolytes within the cell and, if this were to continue indefinitely, osmotic and turgor pressures would fall to zero (Cosgrove, 1985; Schopfer, 2006). The synthesis, assimilation and movement of solutes are then important processes that prevent this situation and thus help maintain cell expansion for long periods. 


\section{Plasmodesmata and their permeability}

In this section, we briefly describe the structure and function of a plasmodesma channel (Fig. 1). We then focus on questions such as how the size of the channel is regulated and why such regulation is important for turgor pressure. Our aim is not to provide a comprehensive discussion about plasmodesmal permeability regulation (see e.g. Benitez-Alfonso et al., 2011; Faulkner and Maule, 2011; Burch-Smith and Zambryski, 2012; Knox and Benitez-Alfonso, 2014; Han and Kim, 2016) but only to draw attention to points relevant to the links between permeability and mechanical forces.

Plasmodesmata encase a tube of endoplasmic reticulum (ER), known as the desmotubule. Plasmodesmata are divided into two types, primary, which form during cytokinesis, and secondary, which form during cell expansion; they may be simple (a single tube), occur in pairs (two neighboring tubes), or be branched (multiple interconnected tubes) (Burch-Smith and Zambryski, 2012). Plasmodesmata may allow the movement of molecules between cells through their central cavity (the cytoplasmic sleeve around the desmotuble) or through the desmotubule (Barton et al., 2011). Symplasmic connectivity has been related to changes in the diameter of the pore, which is related to the size exclusion limit (the size of the largest molecule that freely moves through the pore), to the type of plasmodesma, and to their density. For example, a decrease in green fluorescent protein (GFP) movement was observed in leaves that transitioned from sink to source that coincided with an increase in the formation of branched plasmodesmata (Oparka et al., 1999). Regarding the size of the plasmodesmal channel, it is important to note that the movement of molecules that are small enough to pass through in a passive way, called non-targeted transport, is differentiated from the targeted transport that may involve structural modifications of the channel or of the molecules in movement (Lucas et al., 1995). The molecular size of fluorescent dyes has been used to study symplasmic transport and can be expressed in terms of molecular mass (in $\mathrm{kDa}$ ) or physical size (in $\mathrm{nm}$ ). 5(6)-Carboxyfluorescein (CF) is a dye whose molecular size $(0.61 \mathrm{~nm})$ is considered small enough for it to pass through the plasmodesmal pore (Wang and Fisher, 1994); it is confined to the phloem and its fluorescence pattern is similar to the distribution of $\left[{ }^{14} \mathrm{C}\right]$ sucrose (Grignon et al., 1989). Furthermore, the molecular sizes of sucrose $(0.47 \mathrm{~nm}$ ) (Wang and Fisher, 1994; Terry et al., 1995) and CF are comparable and therefore CF has been used as a fluorescent dye for live imaging of phloem unloading (Oparka et al., 1994).

The observation of movement of fluorescent molecules small enough to pass through plasmodesmata has led to the discovery of symplasmic fields. These can be conceptualized as cells or tissues that are more connected among themselves than with surrounding cells, which implies a certain degree of discontinuity that can be incomplete and modified during development (Gisel et al., 1999). For example, symplasmic fields were inferred in Arabidopsis from the patterns of movement of $1 X_{-}$, $2 \times-$, and $3 \times$-GFP fusions during embryo development, which represented three molecular sizes (i.e. 27, 54, and $81 \mathrm{kDa}$ ) expressed under the SHOOT MERISTEMLESS (STM) promoter (Kim and Zambryski, 2005). At the early heart stage $1 \times_{-}$, and $2 \times-$ GFP could move throughout the whole embryo, but by the late heart stage $2 \times$-GFP could not enter into the cotyledons (Kim et al., 2005). Also at the late heart stage, $2 \times$ - but not $3 \times$-GFP could move into the root tip, thereby suggesting the existence of a third symplasmic field (Kim and
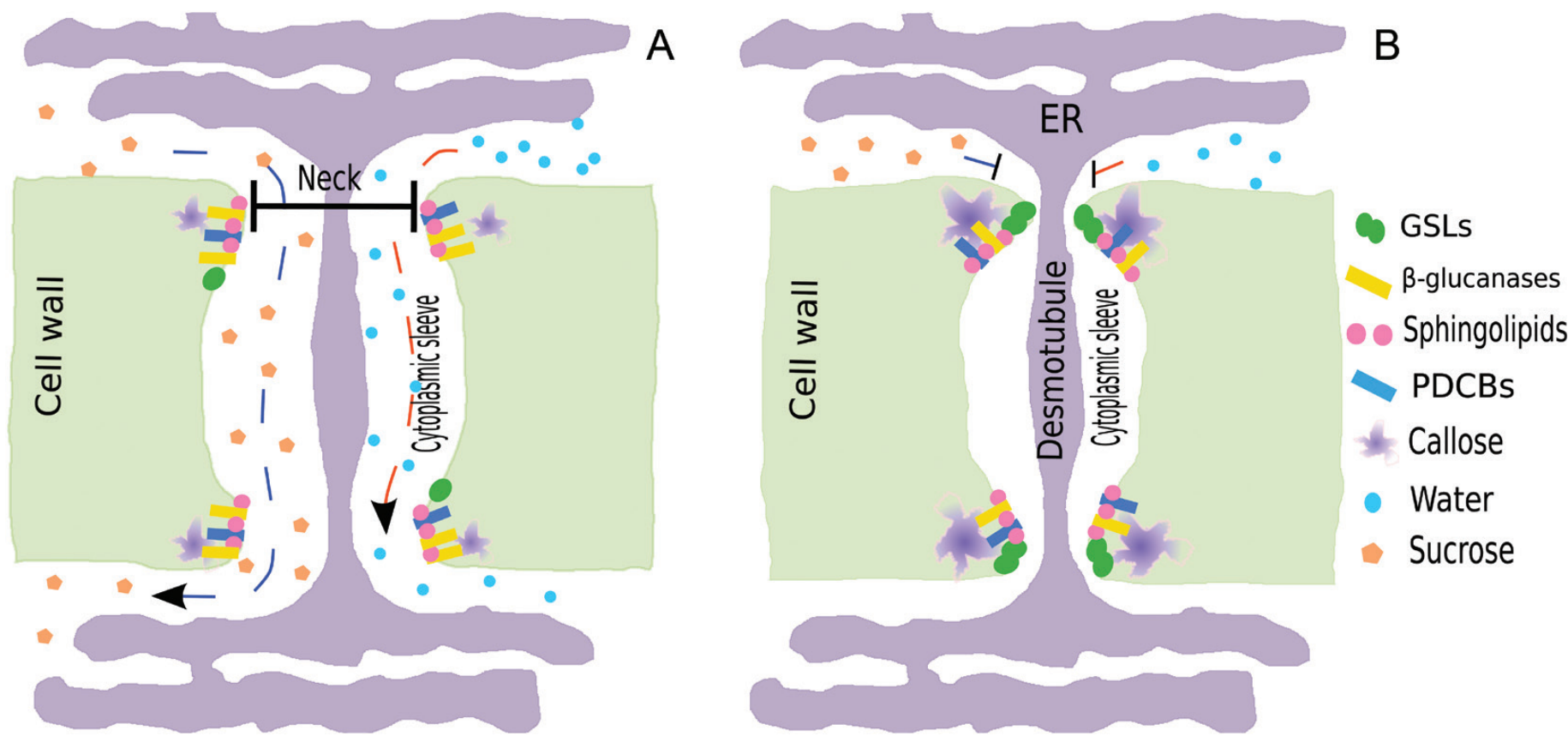

Fig. 1. Schematic representation of the plasmodesmal pore that connects two neighboring cells through their common cell wall. (A) As a result of a lower levels of callose at plasmodesmal necks, the pore is widened and molecules that do not exceed the size of the neck can freely pass. (B) Callose accumulates at plasmodesmal neck thus narrowing the channels and limiting free movement of molecules from one cell to its neighbor. GSL: GLUCAN SYNTHASE-LIKE; PDCB: PLASMODESMATA CALLOSE-BINDING PROTEIN. 
Zambryski, 2005; Kim et al., 2005). These experiments led to the conclusion that plants generate symplasmic fields that can spatiotemporally vary during development.

Plasmodesmal permeability has been related to levels of callose, a $\beta$-glucan that is incorporated into the cell wall at the plasodesmal neck (Drake et al., 1978; Guseman et al., 2010; Vatén et al., 2011; Benitez-Alfonso et al., 2013; Han et al., 2014). GLUCAN SYNTHASE-LIKE (GSL) proteins, also called Callose Synthases (CalS), have been established as the putative family for the synthesis of callose, while the degradation of callose was ascribed to plasmodesmal-localized $\beta-1,3-$ glucanases (PdBGs) (Levy et al., 2007; Guseman et al., 2010). Mutation of the callose synthase GSL 8 was linked to decreased callose levels at the cell plate and at plasmodesmata, defects in cell division, disruption of cellular and tissue patterning, formation of islands of excessive cell proliferation, and cell wall stubs (Chen et al., 2009; Guseman et al., 2010). In the leaves of Arabidopsis, the chor mutant, a weak allele of GSL8, has abnormal stomatal clusters that are assumed to result from the leakage of cell-fate determinants from stomatal lineage cells towards surrounding epidermal cells due to higher plasmodesmal permeability (Guseman et al., 2010). Conversely, a mutation of a $P d B G$ gene would be expected to produce a less permeable plasmodesmal channel and reduce symplasmic movement of molecules. This was reported in pdbg1,2 double mutants, which showed a decreased movement of GFP from the phloem to neighboring tissues in comparison with wild type Arabidopsis roots (Benitez-Alfonso et al., 2013). Moreover, pdbg1,2 double mutant presented formation of lateral root clusters, suggesting that lower permeability of the plasmodesmal channel impaired the specification of lateral root founder cells, probably by altering the movement of cell-specifying molecules (Benitez-Alfonso et al., 2013). In agreement with the expected callose levels of these two mutants, the two studies reported lower (in the GSL8 mutant) and higher callose levels (in pdbg1,2 double mutant) by means of aniline blue staining and immunolocalizations (Guseman et al., 2010; Benitez-Alfonso et al., 2013). In summary, these findings support the idea that some members of the GSL and PdBG families of proteins regulate plasmodesmata opening by modulating callose levels.

What biophysical processes determine permeability of a plasmodesma? Two processes play a role in the movement of molecules in the symplasm: diffusion and advection (Squires and Quake, 2005; Verchot-Lubicz and Goldstein, 2009). On the one hand, diffusion is the net motion of molecules from regions of high to regions of low concentration. As a transport mechanism, diffusion becomes slower and slower as system size increases. Differences in concentration between neighboring cells drive diffusive movement of molecules through the plasmodesmata connecting the two cells. On the other hand, advection corresponds to molecule transport by coherent fluid movement, such as the active cytoplasmic movement known as 'cytoplasmic streaming' or bulk flow in phloem or in xylem, which is generally much more efficient for transport than diffusion (Squires and Quake, 2005; Verchot-Lubicz and Goldstein, 2009). Turgor pressure differences between neighboring cells cause a flow through the plasmodesmata that connect the two cells, driving advective transport of molecules in the direction of the flow. Blake (1978) examined the contributions of fluid properties (viscosity varying with flow rate), of the desmotubule, and of the geometry of the cavity to plasmodesmal permeability and found that the diameter of the neck was the main determinant of permeability, suggesting that changes in neck opening are sufficient to modulate plasmodesma-dependent fluxes. More generally, it is expected that both advection and diffusion are involved in molecular movement through plasmodesmata, with relative weight that depends on the system and process of interest. For instance, phloem loading by sugars through plasmodesmata appears dominated by diffusion in trees and partially involves advection in herbs (Comtet et al., 2017a, b). In the leaf epidermis, no pressure differences are expected between pavement cells, and so non-targeted transport of molecules through plasmodesmata is believed to be diffusion-driven (Crawford and Zambryski, 2001; Schönknecht et al., 2008). In particular, Schönknecht and coworkers (2008) found that GFP movement in tobacco leaves depends exponentially on the inverse of temperature, like an Arrhenius function with a well-defined activation energy, leading them to ascribe movement to diffusion. Nevertheless, the existence of an activation energy means that the process is not simple diffusion-GFP movement would be driven by differences in concentrations between cells and would involve a limiting step (with a specific activation energy), which could be a change in GFP conformation before traversing the plasmodesma. Altogether, the movement of small molecules through the plasmodesma likely involves molecular diffusion and advection due to turgor pressure differences, whereas the movement of large molecules is likely limited by interactions with the channel. Note, however, that more complex processes might lead to transport through plasmodesmata, such as electroosmosis whereby ion movement is driven by electrostatic fields (Squires and Quake, 2005) due, for instance, to differences in membrane potential between neighboring cells.

Plasmodesmal permeability seems to be key to establishing patterns of cell types, which is a necessary process for the evolution of vascular tissues and complex multicellular plants. For example, ectopic expression of callose accumulation prevented intercellular movement of the transcription factor SHORTROOT and the microRNAs miR $165 / 6$ between stele and ground tissues (Vatén et al., 2011). In light of the above, one could hypothesize that the role of plasmodesmal permeability in cell type specification and tissue development had important evolutionary significance. Plasmodesmata evolved several times in the plant lineage. Plasmodesmal permeability in some multicellular green algae, like Bulbochaete hiloensis (Fraser and Gunning, 1969), can also change during ontogeny yielding well-defined symplasmic fields (Raven, 1997; Cook and Graham, 1999; Kwiatkowska, 1999). This suggests, along with other lines of evidence, that plasmodesmata can fulfill similar functions in very diverse groups and that they might have enabled similar functions in early plant evolution (Benítez et al., 2018). For instance, the specification of cell identities dependent on the creation of symplasmic fields may have led to the evolution of vascular tissues that are able to transport nutrients through long distances, which could have been 
instrumental in the expansion of the plant lineage on land (Benítez et al., 2018).

\section{A door closes: plasmodesmal permeability may help build turgor pressure}

The regulation of plasmodesmal permeability can affect the movement and accumulation of solutes and probably, by extension, osmotic pressure. It has been reported that a decrease of the plasmodesmal diameter at the neck is correlated with lower levels of import of ${ }^{14} \mathrm{C}$-labelled sucrose in pea root tips (Schulz, 1995). Due to the role of osmotic pressure in generating turgor, one could expect that plasmodesma-dependent movement of solutes affects turgor pressure and, by extension, cell expansion. Nevertheless, thorough exploration of the effects of plasmodesmal permeability on turgor pressure and cell expansion has not been carried out.

Cotton fibers provide an ideal system to test the role of plasmodesma-dependent effects on turgor pressure and cell elongation. Some experiments have correlated changes in plasmodesmal permeability, phloem unloading of CF, and cell elongation patterns in cotton fibers (Ruan et al., 2001, 2004; Zhang et al., 2017). A cotton fiber is a single trichome-like epidermal cell that elongates about 1000-fold from its initial size, without dividing. CF translocation via the phloem was observed at different times of cotton fiber elongation (Ruan et al., 2001); CF was detected in the phloem of the seed coat of cotton and inside the fibers, except between 10 and 15 days after anthesis during which CF was reported absent from the fibers (Ruan et al., 2001). These results led the authors to hypothesize that CF could no longer enter the fibers due to a closure of the plasmodesmal pore. To test this idea, the authors carried out aniline blue staining and immunolocalization, which showed accumulation of callose at the time of the supposed plasmodesmal closure, and they also reported an increase in expression of a fiber-specific $\beta-1,3$-glucanase at the time of re-opening (Ruan et al., 2004). This time window also coincided with a higher expression of sucrose and potassium transporters that, together, resulted in larger values of osmotic and turgor pressures (Ruan et al., 2001). The closure of plasmodesmata might be necessary for fast elongation of the fibers, by preventing leakage of osmolytes out of the cell and enabling turgor pressure buildup.

The hypothesis that plasmodesmal closure is necessary for the influx of water would be testable by using mutations or treatments that induce abnormal callose accumulation. Some evidence along these lines is provided by the consequences of changes in the composition of plasma membrane as explained hereafter. It has been shown that plasmodesmal membranes are enriched with detergent-insoluble membrane (DIM) components such as sterols and sphingolipids (Grison et al., 2015). There are two proteins that are specifically localized at plasmodesmata, possibly due to their glycophosphatidylinositol anchors-callose-binding protein 1 (PDCB1) and $\beta-1,3-$ glucanase (PdBG2) - whose mutations or misexpression affects callose levels and symplasmic movement of proteins (Levy et al., 2007; Simpson et al., 2009; Benitez-Alfonso et al., 2013).
Moreover, these two proteins are known to specifically bind to DIMs of plasmodesmal membrane. It was recently observed that plasmodesmal permeability is altered when the overall composition of sterols is impaired (Grison et al., 2015). In cotton, fiber length was reduced in plants with downregulation of a putative sterol carrier; these plants showed reduced expression of GhPdBG3-2A/D, a plasmodesmal $\beta-1,3$-glucanase that likely anchors to DIMs, and closed plasmodesmata from 5 to 25 days after anthesis, based on CF assay (Zhang et al., 2017). These results point to the importance of plasmodesmal membranes for proper function of proteins that regulate callose levels. They also support the hypothesis that plasmodesmal permeability may be important for cell elongation, though further experiments would be needed to directly test this hypothesis, for example by manipulating expression of a GSL gene in fibers.

\section{Turgor strikes back: pressure may induce changes in plasmodesmal permeability}

In the previous section we discussed the evidence supporting the hypothesis that plasmodesmal closure is necessary to build turgor pressure during cell elongation. In this section we will present the experiments that suggest that turgor pressure can induce changes in the permeability of plasmodesmata.

The temporal and spatial variations of plasmodesmal permeability, during development and across tissues, raise the question of what factors can regulate the permeability of the channel. Olesen (1979) stained plasmodesmata in leaves of Salsola kali and in roots of Epilobium hirsutum and observed a ring-like structure at the entrance of the channel that led him to hypothesize that plasmodesmata served as 'sphincters with a valve function'. Such a valve function could be mediated by deformation of the desmotubule (Blake, 1978), of the plasma membrane, or of the cell wall. Côté et al. (1987) measured the electric resistance between the vacuoles of two neighboring internodal cells in Chara corallina, and found that the resistance increased significantly when one of the two cells was bathed in a hyperosmotic solution, inducing a turgor pressure of about $0.24 \mathrm{MPa}$ between the two cells. Because the electric conductance between cells is mostly dependent on symplasmic conductivity, they ascribed their observation to the difference in turgor pressure inducing gating of plasmodesmata. Oparka and Prior (1992) conducted a series of experiments with a pressure probe aimed at inducing pressure differentials between pairs of neighboring cells of tobacco trichomes. They punctured a cell to release turgor and also performed the reciprocal experiment by injecting a drop of oil inside to increase the pressure. To test whether there were any changes in the symplasmic connectivity after these changes of pressure, they injected Lucifer Yellow $\mathrm{CH}(\mathrm{LYCH})$ dye in the most apical cell of the trichome and observed its movement between cells. In control treatments, LYCH could move through the cells all the way down towards the base of the trichome. After puncturing one of the trichome cells, they measured turgor pressure of the apical and basal neighbors and found that these other cells also reduced their turgor. Furthermore, the pressure differentials 
found correlated with LYCH movement, namely, LYCH could not enter the cell with the smallest turgor pressure, suggesting that plasmodesmata respond to turgor differentials. This phenomenon was recently modelled by Park et al. (2019) who hypothesized that fast closing of plasmodesmata would be due to higher turgor pressure on one side pressing the endoplasmic reticulum against the cell membrane; using plausible values of physical parameters, they recovered variations of relative permeability with pressure that are in semi-quantitative agreement with experiments.

When plants were treated with osmotic solutions intended to induce plasmolysis, callose levels increased in oat coleoptiles (Drake et al., 1978), plasmodesmal pores narrowed in pea root tips (Schulz, 1995), and symplasmic continuity was disrupted in Egeria densa leaves (Erwee and Goodwin, 1984). However, in the study performed by Schulz (1995), moderate treatments with mannitol that did not plasmolyse cells were shown to transiently increase the size of the plasmodesmal pore and enhance movement of sugars to osmotically stressed tissues. These findings suggest that osmotic treatments can induce two opposite responses on plasmodesmal permeability depending on the intensity of the stress. Schulz (1995) proposed that a transient enhancement of sugar import after a moderate osmotic stress was needed to lower the water potential to prevent plasmolysis. But when the stress is sufficient to plasmolyse the cell, plasmodesmata close, potentially preventing further cytoplasmic leakage. Hence, changes in turgor pressure can affect the permeability of plasmodesmal channels but the evidence suggests that the response is not linear. Further work is needed to fully understand plasmodesmal response to changes in turgor and determine what threshold values of turgor can close the plasmodesmal channel.

An analysis of the biochemical networks that regulate plasmodesmal permeability and turgor pressure reveals factors shared between these two processes. Reactive oxygen species (ROS) have been shown to play an important role in cell expansion. ROS production in the apoplast is linked to the loosening of cell walls through cleavage by hydroxyl radicals of polysaccharides such as pectins and homogalacturans (Fry et al., 2001; Schopfer et al., 2001), though ROS may also lead to cell-wall stiffening by creating new cross-links (Passardi et al., 2004). Accordingly, ROS regulate turgor pressure through their effects on cell wall relaxation. In addition, ROS accumulation in roots of Arabidopsis and cucumber has been related to intracellular trafficking of aquaporins and a reduction in water transport, adding another layer to the relationship between ROS and turgor (Lee et al., 2004; Boursiac et al., 2008). ROS seem to also regulate plasmodesmal permeability as their accumulation is correlated to increases in callose deposition and plasmodesmal closure (Cui and Lee, 2016). CF movement was restricted after mechanical wounding and $\mathrm{H}_{2} \mathrm{O}_{2}$ spraying of Arabidopsis plants (Cui and Lee, 2016). Furthermore, ROS production seems to be downstream of mechanical signals. Mechanically wounded Arabidopsis plants that were pretreated with ascorbic acid-a reducing agent intended to block ROS production-did not show the mechanically induced plasmodesmal closure that was observed in non-pretreated plants (Cui and Lee, 2016).
The CalS 8 gene seems to mediate the response to mechanical wounding via extracellular ROS, as a cals 8 mutant was defective in both ROS- and mechanical wounding-induced plasmodesmal regulation (Cui and Lee, 2016). The production of ROS species, therefore, seems to be an important factor linking mechanical signals coming from cell expansion and wounding to responses like plasmodesmal permeability and turgor pressure regulation.

$\mathrm{Ca}^{2+}$ is a structural component of the cell wall and acts as a second messenger responding to environmental signals. Experimental evidence suggests that $\mathrm{Ca}^{2+}$ might play a role in the regulation of callose synthesis through its interactions with annexins (ANNs), calmodulins (CaM), and caltireticulins. ANNs are membrane-bound proteins with GTPase activity dependent on $\mathrm{Ca}^{2+}$. They are expressed in elongating cotton fibers and were found to potentially interact with callose synthases (Andrawis et al., 1993; Shin and Brown, 1999). Also a group of proteins that resemble plant and animal ANNs were related to the activity of callose synthase in vitro in a $\mathrm{Ca}^{2+}$-dependent manner (Andrawis et al., 1993). ANNs seem to respond to accumulation of ROS in Arabidopsis roots by regulating cytoplasmic $\mathrm{Ca}^{2+}$ through permeable channels, thus serving as a link between ROS and $\mathrm{Ca}^{2+}$ (Richards et al., 2014). CaM are $\mathrm{Ca}^{2+}$ sensors that show conserved regulatory activity in eukaryotic cells. In root hairs of Arabidopsis, ROS and $\mathrm{Ca}^{2+}$ are involved in a positive feedback loop. First, ROS accumulation activates calcium influx (Foreman et al., 2003) that, in turn, activates CaM, which then targets NADPH-dependent production of ROS (Harding et al., 1997; Takeda et al., 2008). In cotton fibers, overexpression of a cotton calmodulin, GhCaM7, leads to higher concentrations of ROS and promotes early fiber elongation, while exogenous $\mathrm{H}_{2} \mathrm{O}_{2}$ application in WT plants enhanced the expression of GhCaM7 as well as early fiber elongation (Tang et al., 2014). The calcium-binding protein calreticulin was found to be located in ER of plasmodesmal channels (Baluska et al., 1999), there is a correlation between calreticulin and callose accumulation at plasmodesmata (Bilska and Sowinski, 2010), and calreticulin interacts with a glucosaminyl transferase-like (GnTL), which co-localizes with PDCB (Zalepa-King and Citovsky, 2013). This suggests that calreticulin may interact with enzymes that regulate callose levels at plasmodesmata.

$\mathrm{Ca}^{2+}$ might also close plasmodesmata in a calloseindependent manner. This hypothesis stems from the observation that increases in cytoplasmic $\mathrm{Ca}^{2+}$ induced by microinjection of mastoparan in staminal hair cells of Setcreasea purpurea transiently close plasmodesmata within seconds, with plasmodesmata remaining closed for less than a minute (Tucker and Boss, 1996). Calreticulin might associate with acto-myosin and centrin at plasmodesmal necks to translate calcium fluctuations into transient contractions of the pore (Sager and Lee, 2014), which would constitute a callose-independent form of plasmodesmal permeability regulation.

Put together, experimental evidence discussed here suggests that the biochemical interactions that connect turgor pressure, plasmodesmal permeability, and cell elongation constitute a tightly coupled network (Fig. 2). 


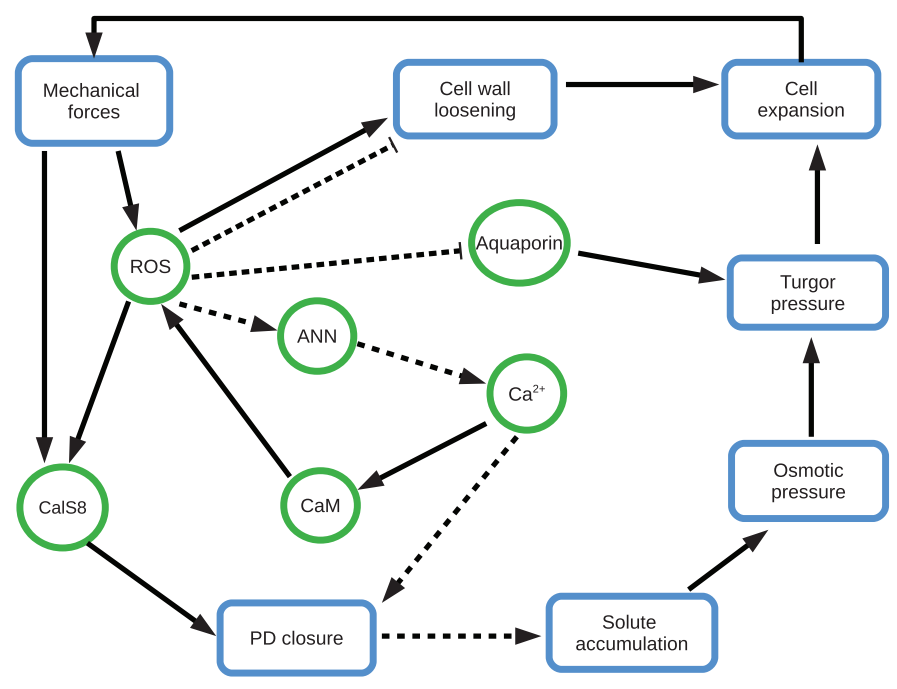

Fig. 2. Network of interactions between biochemical, mechanical factors, and processes that connect plasmodesmal permeability, turgor pressure, and cell wall expansion. Blue rectangles represent processes, and red ovals represent molecules. Arrows correspond to upregulation, T-ending lines to down-regulation; dotted arrows and lines indicate hypothetical interactions.

\section{Questions and future directions}

We have summarized available evidence suggesting that plasmodesmal permeability and turgor pressure are linked and put forward two hypotheses about how these two factors might interact. Namely, plasmodesmata permeability may contribute to osmolyte accumulation and hence turgor-driven cell expansion; turgor, in turn, can also affect plasmodesmal permeability, possibly through mechanically induced ROS accumulation and calcium signaling or directly by pushing the endoplasmic reticulum against the plasma membrane. However, there are many gaps to fill. In this section we discuss particular questions that need to be addressed.

Experimental data support the assumption that narrowing the neck of the pore is necessary for building turgor pressure (Ruan et al., 2001, 2004); however, it is important to consider that symplasmic connectivity can also vary due to changes in plasmodesmal number, for instance by de novo formation of branched plasmodesmata (Oparka et al., 1999), as observed during cotton fiber elongation (Ruan et al., 2001). It is therefore necessary to investigate whether turgor is affected because of changes in diameter of simple plasmodesmata, formation of branched plasmodesmata, or a combination thereof. These possibilities also apply to the effects of turgor pressure on plasmodesmal permeability because loosening of the cell wall might be involved in the formation of branched plasmodesmata (Ehlers and van Bel, 2010). Using 3D reconstructions from transmission electron microscopy (Nicolas et al., 2017), it is now possible to examine the physical structure of plasmodesmata and examine their nature, helping to clarify the aforementioned issues.

It would be interesting to test whether changes in turgor pressure induced by chemical treatments can alter the patterns of non-targeted symplasmic movement. Even considering the evidence provided by Schulz (1995) of osmotic treatments altering plasmodesmal diameter and sucrose unloading, actual turgor pressure was not measured in this study to corroborate this link. This is particularly important because osmotic treatments can trigger many physiological responses in plants. It is then necessary to measure turgor pressure in osmotic treatments and verify whether those osmotic stress-induced values can alter callose deposition and symplasmic movement of molecules. These experiments can also be applied not only to the non-targeted but also to the targeted movement of transcription factors that are known to control the structure of plasmodesmata, e.g. KNOTTED1 (KN1) (Xu et al., 2011). Such measurements of turgor pressure might be eased by indentation-based approaches that were recently developed (Beauzamy et al., 2015; Weber et al., 2015).

Osmotic stress can either increase or decrease permeability depending on intensity (Schulz, 1995), which means that the response of plasmodesmata to changes in turgor pressure may be non-linear. In this context, it would be interesting to test how much turgor pressure needs to drop to activate the callosedependent closure of plasmodesmata. Oparka and Prior (1992) found that a pressure differential of $300 \mathrm{kPa}$ prevented symplasmic movement of $\mathrm{LYCH}$, but it is unknown whether this response is mediated by deposition of callose at plasmodesmal necks. An approach combining chemical treatments, turgor measurements, the observation of symplasmic movement of fluorescent molecules, and mathematical or computational models can help elucidate this question. Computational models are particularly useful to provide mechanistic explanations in processes that are based on non-linear interactions and to elucidate the relative contribution of each of its components. As we propose in Fig. 2, there are many factors and processes connecting turgor and plasmodesmal permeability of different nature (e.g. mechanical, molecular), with different time responses (seconds, minutes, hours), and that seem to interact in non-linear ways, which could be integrated together in a computational model to put forward testable predictions. For example, a computational model that studies the changes in callose levels to turgor pressure could help find what type of function best describes this response and how this, in turn, could affect turgor.

Studies on the effects of turgor pressure on plasmodesmal permeability have been carried out with different systems. Cotton fibers would be appropriate to perform these studies in a single system, though it may not be the easiest choice because of its allopolyploidy and, until recently, because of the low efficiency of genetic transformation of cotton (Chen et al., 2017). Nevertheless, the CRISPR/Cas9 system is becoming an efficient strategy for targeted mutagenesis of cotton and could help generate mutations of proteins that regulate callose levels to see how they affect cotton fiber elongation (Chen et al., 2017; Gao et al., 2017). An alternative system could be trichoblasts (and/or trichomes) of Arabidopsis, which are also single cells, and mutants of genes that code for proteins that regulate callose levels at plasmodesmata are readily available. To test the role of plasmodesmata in the elongation of trichoblasts, it would be necessary to follow the approaches of Ruan and coworkers $(2001,2004)$ for cotton fibers, evaluating the expression of GSLs and/or PdGBs and then testing the effects of mutations of these genes on root hair elongation. 


\section{Conclusions}

Turgor pressure and plasmodesmal permeability can affect each other during plant development. Plasmodesmata control the content of osmolytes and water within the cell and turgor pressure can affect the levels of callose levels and the non-targeted movement of molecules. These interactions have important consequences for plant growth and development. Because plasmodesmal permeability helps establish patterns of cell types, for instance during differentiation of stomata or of vascular tissues, the study of how turgor pressure regulates plasmodesmal permeability can improve our understanding of how multicellular plants, and notably vascular plant, evolved. In this review, we have compiled the evidence that supports our hypothesis and we have identified some of the key questions that remain open in an attempt to bring attention to the subject.

\section{Acknowledgements}

This work was supported by CONACYT and by Institut Universitaire de France.

\section{References}

Andrawis A, Solomon M, Delmer DP. 1993. Cotton fiber annexins: a potential role in the regulation of callose synthase. The Plant Journal $\mathbf{3}$, 763-772.

Baluska F, Samaj J, Napier R, Volkmann D. 1999. Maize calreticulin localizes preferentially to plasmodesmata in root apex. The Plant Journal 19, 481-488.

Barton DA, Cole L, Collings DA, Liu DY, Smith PM, Day DA, Overall RL. 2011. Cell-to-cell transport via the lumen of the endoplasmic reticulum. The Plant Journal 66, 806-817.

Beauzamy L, Derr J, Boudaoud A. 2015. Quantifying hydrostatic pressure in plant cells by using indentation with an atomic force microscope. Biophysical Journal 108, 2448-2456.

Beauzamy L, Nakayama N, Boudaoud A. 2014. Flowers under pressure: ins and outs of turgor regulation in development. Annals of Botany 114, 1517-1533.

Benítez M, Hernández-Hernández V, Newman SA, Niklas KJ. 2018. Dynamical patterning modules, biogeneric materials, and the evolution of multicellular plants. Frontiers in Plant Sciences 9, 871.

Benitez-Alfonso Y, Faulkner C, Pendle A, Miyashima S, Helariutta Y, Maule A. 2013. Symplastic intercellular connectivity regulates lateral root patterning. Developmental Cell 26, 136-147.

Benitez-Alfonso Y, Jackson D, Maule A. 2011. Redox regulation of intercellular transport. Protoplasma 248, 131-140.

Bilska A, Sowinski P. 2010. Closure of plasmodesmata in maize (Zea mays) at low temperature: a new mechanism for inhibition of photosynthesis. Annals of Botany 106, 675-686.

Blake JR. 1978. On the hydrodynamics of plasmodesmata. Journal of Theoretical Biology 74, 33-47.

Boursiac Y, Prak S, Boudet J, Postaire O, Luu DT, Tournaire-Roux C, Santoni V, Maurel C. 2008. The response of Arabidopsis root water transport to a challenging environment implicates reactive oxygen species- and phosphorylation-dependent internalization of aquaporins. Plant Signaling \& Behavior 3, 1096-1098.

Buckley TN. 2005. The control of stomata by water balance. New Phytologist 168, 275-292.

Burch-Smith TM, Zambryski PC. 2012. Plasmodesmata paradigm shift: regulation from within versus without. Annual Review of Plant Biology 63, 239-260.
Chen X, Lu X, Shu N, Wang S, Wang J, Wang D, Guo L, Ye W. 2017. Targeted mutagenesis in cotton (Gossypium hirsutum L.) using the CRISPR/ Cas9 system. Scientific Reports 7, 44304.

Chen XY, Lin L, Lee E, Han X, Rim Y, Chu H, Kim SW, Sack F, Kim JY. 2009. The Arabidopsis callose synthase gene GSL8 is required for cytokinesis and cell patterning. Plant Physiology 150, 105-113.

Comtet J, Jensen KH, Turgeon R, Stroock AD, Hosoi AE. 2017 a. Passive phloem loading and long-distance transport in a synthetic tree-ona-chip. Nature Plants 3, 17032.

Comtet J, Turgeon R, Stroock AD. 2017b. Phloem loading through plasmodesmata: a biophysical analysis. Plant Physiology 175, 904-915.

Cook M, Graham L. 1999. Evolution of plasmodesmata. In: Van Bel AJE, van Kesteren WJP, eds. Plasmodesmata. Structure, function, role in cell communication. Berlin, Heidelberg: Springer, 101-117.

Cosgrove DJ. 1985. Cell wall yield properties of growing tissue: evaluation by in vivo stress relaxation. Plant Physiology 78, 347-356.

Cosgrove DJ. 2005. Growth of the plant cell wall. Nature Reviews. Molecular Cell Biology 6, 850-861.

Côté R, Thain JF, Fensom DS. 1987. Increase in electrical resistance of plasmodesmata of Chara induced by an applied pressure gradient across nodes. Canadian Journal of Botany 65, 509-511.

Crawford KM, Zambryski PC. 2001. Non-targeted and targeted protein movement through plasmodesmata in leaves in different developmental and physiological states. Plant Physiology 125, 1802-1812.

Cui W, Lee JY. 2016. Arabidopsis callose synthases CalS1/8 regulate plasmodesmal permeability during stress. Nature Plants 2, 16034.

De Storme N, Geelen D. 2014. Callose homeostasis at plasmodesmata: molecular regulators and developmental relevance. Frontiers in Plant Science 5, 138.

Drake GA, Carr DJ, Anderson WP. 1978. Plasmolysis, plasmodesmata, and the electrical coupling of at coleoptile cells. Journal of Experimental Botany 29, 1205-1214.

Ehlers K, van Bel AJ. 2010. Dynamics of plasmodesmal connectivity in successive interfaces of the cambial zone. Planta 231, 371-385.

Erwee MG, Goodwin PB. 1984. Characterization of the Egeria densa leaf symplast: response to plasmolysis, deplasmolysis and to aromatic amino acids. Protoplasma 122, 162-168.

Faulkner C, Maule A. 2011. Opportunities and successes in the search for plasmodesmal proteins. Protoplasma 248, 27-38.

Foreman J, Demidchik V, Bothwell JH, et al. 2003. Reactive oxygen species produced by NADPH oxidase regulate plant cell growth. Nature 422, 442-446.

Fraser TW, Gunning BE. 1969. The ultrastructure of plasmodesmata in the filamentous green alga, Bulbochaete hiloensis (Nordst.) tiffany. Planta 88, 244-254.

Fry SC, Dumville JC, Miller JG. 2001. Fingerprinting of polysaccharides attacked by hydroxyl radicals in vitro and in the cell walls of ripening pear fruit. The Biochemical Journal 357, 729-737.

Gao W, Long L, Tian X, Xu F, Liu J, Singh PK, Botella JR, Song C. 2017. Genome editing in cotton with the CRISPR/Cas9 system. Frontiers in Plant Sciences 8, 1364.

Gisel A, Barella S, Hempel FD, Zambryski PC. 1999. Temporal and spatial regulation of symplastic trafficking during development in Arabidopsis thaliana apices. Development 126, 1879-1889.

Grignon N, Touraine B, Durand M. 1989. 6(5)Carboxyfluorescein as a tracer of phloem sap translocation. American Journal of Botany $\mathbf{7 6}$, 871-877.

Grison MS, Brocard L, Fouillen L, et al. 2015. Specific membrane lipid composition is important for plasmodesmata function in Arabidopsis. The Plant Cell 27, 1228-1250.

Guseman JM, Lee JS, Bogenschutz NL, Peterson KM, Virata RE, Xie B, Kanaoka MM, Hong Z, Torii KU. 2010. Dysregulation of cell-tocell connectivity and stomatal patterning by loss-of-function mutation in Arabidopsis chorus (glucan synthase-like 8). Development 137, 1731-1741.

Hamant O, Traas J. 2009. The mechanics behind plant development. New Phytologist 185, 369-385.

Han X, Hyun TK, Zhang M, Kumar R, Koh EJ, Kang BH, Lucas WJ, Kim JY. 2014. Auxin-callose-mediated plasmodesmal gating is essential 
for tropic auxin gradient formation and signaling. Developmental Cell $\mathbf{2 8}$ 132-146.

Han X, Kim JY. 2016. Integrating hormone- and micromolecule-mediated signaling with plasmodesmal communication. Molecular Plant 9, 46-56.

Harding SA, Oh SH, Roberts DM. 1997. Transgenic tobacco expressing a foreign calmodulin gene shows an enhanced production of active oxygen species. The EMBO Journal 16, 1137-1144.

Jaffe MJ, Huberman M, Johnson J, Telewski FW. 1985. Thigmomorphogenesis: the induction of callose formation and ethylene evolution by mechanical perturbation in bean stems. Physiologia Plantarum 64, 271-279.

Kim I, Kobayashi K, Cho E, Zambryski PC. 2005. Subdomains for transport via plasmodesmata corresponding to the apical-basal axis are established during Arabidopsis embryogenesis. Proceedings of the National Academy of Sciences, USA 102, 11945-11950.

Kim I, Zambryski PC. 2005. Cell-to-cell communication via plasmodesmata during Arabidopsis embryogenesis. Current Opinion in Plant Biology 8, 593-599.

Knox JP, Benitez-Alfonso Y. 2014. Roles and regulation of plant cell walls surrounding plasmodesmata. Current Opinion in Plant Biology 22, 93-100.

Kroeger JH, Zerzour R, Geitmann A. 2011. Regulator or driving force? The role of turgor pressure in oscillatory plant cell growth. PLoS One $\mathbf{6}$, e18549.

Kwiatkowska M. 1999. Plasmodesmal coupling and cell differentiation in algae. In: Van Bel AJE, van Kesteren WJP, eds. Plasmodesmata. Structure, function, role in cell communication. Berlin, Heidelberg: Springer, 205-224.

Lee JY, Wang X, Cui W, et al. 2011. A plasmodesmata-localized protein mediates crosstalk between cell-to-cell communication and innate immunity in Arabidopsis. The Plant Cell 23, 3353-3373.

Lee SH, Singh AP, Chung GC. 2004. Rapid accumulation of hydrogen peroxide in cucumber roots due to exposure to low temperature appears to mediate decreases in water transport. Journal of Experimental Botany 55, 1733-1741.

Levy A, Erlanger M, Rosenthal M, Epel BL. 2007. A plasmodesmataassociated $\beta$-1,3-glucanase in Arabidopsis. The Plant Journal 49, 669-682.

Lucas M, Kenobi K, von Wangenheim D, et al. 2013. Lateral root morphogenesis is dependent on the mechanical properties of the overlaying tissues. Proceedings of the National Academy of Sciences, USA 110, 5229-5234.

Lucas WJ, Bouché-Pillon S, Jackson DP, Nguyen L, Baker L, Ding B, Hake S. 1995. Selective trafficking of KNOTTED1 homeodomain protein and its mRNA through plasmodesmata. Science 270, 1980-1983.

Lucas WJ, Lee JY. 2004. Plasmodesmata as a supracellular control network in plants. Nature Reviews. Molecular Cell Biology 5, 712-726.

Moulia B. 2013. Plant biomechanics and mechanobiology are convergent paths to flourishing interdisciplinary research. Journal of Experimental Botany 64, 4617-4633.

Münch E. 1930. Die Stoffbewegungen in der Pflanze. Jena: Gustav Fischer. Nicolas WJ, Grison MS, Trépout S, Gaston A, Fouché M, Cordelières FP, Oparka K, Tilsner J, Brocard L, Bayer EM. 2017. Architecture and permeability of post-cytokinesis plasmodesmata lacking cytoplasmic sleeves. Nature Plants 3, 17082.

Niklas KJ, Spatz HC. 2012. Plant physics. Chicago: University of Chicago Press, 71-103.

Olesen P. 1979. The neck constriction in plasmodesmata: evidence for a peripheral sphincter-like structure revealed by fixation with tannic acid. Planta 144, 349-358.

Oparka KJ, Duckett CM, Prior DAM, Fisher DB. 1994. Real-time imaging of phloem unloading in the root tip of Arabidopsis. The Plant Journal 6, 759-766.

Oparka KJ, Prior DAM. 1992. Direct evidence for pressure-generated closure of plasmodesmata. The Plant Journal 2, 741-750.

Oparka KJ, Roberts AG, Boevink P, Santa Cruz S, Roberts I, Pradel KS, Imlau A, Kotlizky G, Sauer N, Epel B. 1999. Simple, but not branched, plasmodesmata allow the nonspecific trafficking of proteins in developing tobacco leaves. Cell 97, 743-754.

Park K, Knoblauch J, Oparka K, Jensen K. 2019. Controlling intercellular flow through mechanosensitive plasmodesmata nanopores. Nature Communications 10, 3564
Passardi F, Penel C, Dunand C. 2004. Performing the paradoxical: how plant peroxidases modify the cell wall. Trends in Plant Science 9, 534-540.

Péret B, Li G, Zhao J, et al. 2012. Auxin regulates aquaporin function to facilitate lateral root emergence. Nature Cell Biology 14, 991-998.

Raven JA. 1997. Miniview: multiple origins of plasmodesmata. European Journal of Phycology 32, 95-101.

Richards SL, Laohavisit A, Mortimer JC, Shabala L, Swarbreck SM, Shabala S, Davies JM. 2014. Annexin 1 regulates the $\mathrm{H}_{2} \mathrm{O}_{2}$-induced calcium signature in Arabidopsis thaliana roots. The Plant Journal 77, 136-145.

Rinne PL, van der Schoot C. 1998. Symplasmic fields in the tunica of the shoot apical meristem coordinate morphogenetic events. Development 125, 1477-1485.

Ruan YL, Llewellyn DJ, Furbank RT. 2001. The control of single-celled cotton fiber elongation by developmentally reversible gating of plasmodesmata and coordinated expression of sucrose and $\mathrm{K}^{+}$transporters and expansin. The Plant Cell 13, 47-60.

Ruan YL, Xu SM, White R, Furbank RT. 2004. Genotypic and developmental evidence for the role of plasmodesmatal regulation in cotton fiber elongation mediated by callose turnover. Plant Physiology 136, 4104-4113.

Sager R, Lee JY. 2014. Plasmodesmata in integrated cell signalling: insights from development and environmental signals and stresses. Journa of Experimental Botany 65, 6337-6358.

Schmalstig JG, Cosgrove DJ. 1990. Coupling of solute transport and cel expansion in pea stems. Plant Physiology 94, 1625-1633.

Schönknecht G, Brown JE, Verchot-Lubicz J. 2008. Plasmodesmata transport of GFP alone or fused to potato virus X TGBp1 is diffusion driven. Protoplasma 232, 143-152.

Schopfer P. 2006. Biomechanics of plant growth. American Journal of Botany 93, 1415-1425.

Schopfer P, Plachy C, Frahry G. 2001. Release of reactive oxygen intermediates (superoxide radicals, hydrogen peroxide, and hydroxyl radicals) and peroxidase in germinating radish seeds controlled by light, gibberellin, and abscisic acid. Plant Physiology 125, 1591-1602.

Schulz A. 1995. Plasmodesmal widening accompanies the short-term increase in symplasmic phloem unloading in pea root tips under osmotic stress. Protoplasma 188, 22-37.

Shin H, Brown RM Jr. 1999. GTPase activity and biochemical characterization of a recombinant cotton fiber annexin. Plant Physiology $\mathbf{1 1 9}$ 925-934.

Simpson C, Thomas C, Findlay K, Bayer E, Maule AJ. 2009. An Arabidopsis GPI-anchor plasmodesmal neck protein with callose binding activity and potential to regulate cell-to-cell trafficking. The Plant Cell $\mathbf{2 1}$, 581-594.

Squires TM, Quake SR. 2005. Microfluidics: Fluid physics at the nanoliter scale. Reviews of Modern Physics 77, 977.

Takeda S, Gapper C, Kaya H, Bell E, Kuchitsu K, Dolan L. 2008. Loca positive feedback regulation determines cell shape in root hair cells. Science 319, 1241-1244.

Tang W, Tu L, Yang X, Tan J, Deng F, Hao J, Guo K, Lindsey K, Zhang X. 2014. The calcium sensor GhCaM7 promotes cotton fiber elongation by modulating reactive oxygen species (ROS). New Phytologist 202 , 509-520.

Terry BR, Matthews EK, Haseloff J. 1995. Molecular characterisation of recombinant green fluorescent protein by fluorescence correlation microscopy. Biochemical and Biophysical Research Communications 217, 21-27.

Tucker EB, Boss WF. 1996. Mastoparan-induced intracellular $\mathrm{Ca}^{2+}$ fluxes may regulate cell-to-cell communication in plants. Plant Physiology 111, 459-467.

Vatén A, Dettmer J, Wu S, et al. 2011. Callose biosynthesis regulates symplastic trafficking during root development. Developmental Cell 21, 1144-1155.

Verchot-Lubicz J, Goldstein RE. 2009. Cytoplasmic streaming enables the distribution of molecules and vesicles in large plant cells. Protoplasma 240, 99-107.

Vermeer JE, von Wangenheim D, Barberon M, Lee Y, Stelzer EH, Maizel A, Geldner N. 2014. A spatial accommodation by neighboring cells is required for organ initiation in Arabidopsis. Science 343, 178-183.

Wang L, Li XR, Lian H, Ni DA, He YK, Chen XY, Ruan YL. 2010. Evidence that high activity of vacuolar invertase is required for cotton fiber 
and Arabidopsis root elongation through osmotic dependent and independent pathways, respectively. Plant Physiology 154, 744-756.

Wang N, Fisher DB. 1994. The use of fluorescent tracers to characterize the post-phloem transport pathway in maternal tissues of developing wheat grains. Plant Physiology 104, 17-27.

Weber A, Braybrook S, Huflejt M, Mosca G, Routier-Kierzkowska AL, Smith RS. 2015. Measuring the mechanical properties of plant cells by combining micro-indentation with osmotic treatments. Journal of Experimental Botany, 66, 3229-3241.
Xu XM, Wang J, Xuan Z, Goldshmidt A, Borrill PG, Hariharan N, Kim JY, Jackson D. 2011. Chaperonins facilitate KNOTTED1 cell-to-cell trafficking and stem cell function. Science 333, 1141-1144.

Zalepa-King L, Citovsky V. 2013. A plasmodesmal glycosyltransferaselike protein. PLoS One 8, e58025.

Zhang Z, Ruan YL, Zhou N, Wang F, Guan X, Fang L, Shang X, Guo W, Zhu S, Zhang T. 2017. Suppressing a putative sterol carrier gene reduces plasmodesmal permeability and activates sucrose transporter genes during cotton fiber elongation. The Plant Cell 29, 2027-2046. 